\title{
Mild myotonic dystrophy is associated with memory impairment in the context of normal general intelligence
}

\author{
J S Rubinsztein, D C Rubinsztein, P J McKenna, S Goodburn, A J Holland
}

\begin{abstract}
Previous investigations of cognitive function in myotonic dystrophy (DM) suggested that DM subjects have impairment of general intelligence and severe defects in executive function. These studies had to neglect patients with mild clinical disease, since they were generally performed before the availability of DNA testing for DM. We have investigated a group of milder DM cases (defined both by their degree of disability and CTG expansion size) and have not found any defect in general intelligence using the National Adult Reading Test, Standard Progressive Matrices, and Mini-Mental State examination. Three executive function tests were used. The cognitive estimates test and the Wisconsin Card Sorting Test showed tendencies towards impairment, which were of borderline significance, but the verbal fluency task was performed normally. However, in the face of normal general intelligence, mild DM subjects as a group show impairment of memory function. This suggests that memory function may be more sensitive to small CTG repeat expansions than general intelligence.

( $($ Med Genet 1997;34:229-233)
\end{abstract}

Keywords: myotonic dystrophy; trinucleotide repeat; intelligence; memory and executive function.

Myotonic dystrophy (DM) is the commonest form of adult muscular dystrophy, with a population incidence of $1: 8000 .{ }^{1}$ This progressive multisystem disease is inherited in an autosomal dominant manner and is associated with an abnormal expansion of a (CTG)n repeat in the 3' untranslated region of a protein kinase gene on chromosome 19. This (CTG)n stretch is polymorphic in the general population where it varies from five to 37 repeats. DM affected subjects have more than 50 repeats and increasing repeat length correlates with increasing severity of the disease and decreasing age of onset of symptoms. ${ }^{2}{ }^{3}$ The number of repeats associated with disease chromosomes tends to increase when they are transmitted, leading to a general decrease in the age of onset of the disease in successive generations, a phenomenon called anticipation.

$\mathrm{DM}$ is primarily considered as a neurological disease characterised by myotonia and muscle weakness. However, it is clear that this mutation has profound effects on cognition in certain cases. For instance, Harper ${ }^{4}$ showed that in the congenital form of DM (that is, onset at or around birth) the mean IQ was 66 . Other studies have suggested that a downward shift in the IQ curve may be a general feature. ${ }^{56}$

Other facets of cognitive function have been studied in DM with inconsistent results. For instance, memory was reported to be normal in DM by Portwood et al but Chang et al noted a visual memory abnormality. Executive function, which is defined as the ability to think abstractly and to plan, initiate, sequence, monitor, and stop complex behaviour, has been reported to be markedly impaired in a study of 17 DM subjects by Woodward et al. ${ }^{5}$ They performed the category test and the Wisconsin Card Sorting Test and found scores in the brain damaged range for $77 \%$ and $71 \%$ of DM cases, respectively. ${ }^{5}$

It is unclear if some congenital or very severe cases were primarily responsible for the excess of cases with general and specific cognitive impairments in previous studies. An ascertainment bias towards the more severe forms of the disease was almost an inevitable consequence in studies of DM before the mutation was characterised, as many cases of DM with minimal symptoms would have been excluded from earlier studies using strict diagnostic inclusion criteria. The elucidation of the molecular basis for the clinical heterogeneity in DM has made it clear that DM patients should probably be investigated in subgroups, since the adverse consequences of the disease may be restricted to patients who have CTG expansions in particular size ranges. In order to investigate whether cognitive impairment is a feature in DM cases without severe peripheral neurological disease, we have specifically excluded all congenital cases from our analysis. This study focuses on general cognitive function, memory, and executive function.

\section{Subjects and methods}

ASCERTAINMENT

All DM subjects who had a DNA diagnosis of DM established in this Genetics Diagnostic Laboratory were initially considered for inclusion. Subjects had to be between the ages of 18 and 70 years and live in East Anglia. Subjects were excluded if the subject had symptoms from birth (congenital onset of DM) or other neurological disease affecting muscle or cognitive function. Permission was sought from the responsible clinical geneticist/genetic nurse specialist as well as from the general practioner before contacting the patient. A further set of subjects was excluded at the request of their 
clinicians. Reasons for exclusion were: a recent termination of pregnancy, a recent family death, and a third trimester pregnancy. Two subjects were excluded because of current adverse social circumstances and another at the request of a GP because of postnatal depression. After exclusion criteria were applied, 21/57 subjects with DM refused or did not reply to a letter requesting their participation. After these exclusion criteria were applied, only subjects who agreed to participate were contacted. Thirty six subjects with DM from 26 unrelated families took part.

By the selection of subjects from the clinical genetics service rather than the neurological clinic patients, we hoped to include subjects with the full range of disability, rather than only those with severe impairment. This clinical genetics department has made a concerted effort to trace families with DM in order to offer molecular diagnosis to all members, so we found that subjects with mild disability were seen. Ascertainment towards mild disease was considered preferable as we wished to exclude severely learning disabled subjects so that a cognitive assessment using standardised instruments could be used. Subjects were seen at home in all cases except one. The clinical investigator was blind to the triplet repeat length of all subjects.

\section{INSTRUMENTS USED}

(1) A standard questionnaire was administered to obtain the subject's age, length of illness, maternal or paternal inheritance of disease, and age of onset of illness. The muscle weakness or myotonia or both as first noted by the patient defined the onset of the disease, as in Hunter et $a l^{2}{ }^{2}$ In addition, questions were asked to elicit other medical conditions, current medication, schooling, and employment record.

(2) Muscle strength was evaluated using the Medical Research Council examination technique. ${ }^{8}$ We were not aiware of any questionnaire that determined the degree of functional disability in this type of disorder, so the following set of questions was devised by JSR to ascertain this parameter. (i) Are you able to walk up 15 stairs unaided? (ii) Are you able to ride a bicycle? (iii) Are you able to open a jar easily? (iv) Are you able to lift heavy parcels? (A standard example of four full shopping bags was given.) (v) Are you able to lift yourself out of the bath? (vi) Are you able to lift your head off the bed if you were lying flat on it (without turning on the side)? This was used to group subjects into groups of mild (one to two negative replies), moderate (three to four negative replies), and severe (five to six negative replies). There were 22 subjects $(63 \%)$ with mild functional disability, seven with moderate disability $(19 \%)$, and seven with severe disability (19\%).

(3) A cognitive assessment was performed using the following tests.

General intelligence was assessed using: National Adult Reading Test (NART), ${ }^{9}$ a test which estimates premorbid IQ; Standard Progressive Matrices, ${ }^{10}$ an untimed test of current general intelligence, suitable for subjects who may have muscle weakness that would affect writing or speed of task performance. This test has been extensively used and normative data are available. In one subject who performed very poorly, a coloured progressive matrices was performed to obtain a more accurate score; the Mini-Mental State Examination (MMSE), ${ }^{11}$ a screening test for general intellectual impairment that covers orientation, attention, memory, language, and praxic skills.

Memory was tested using the Rivermead Behavioural Memory Test (RBMT). ${ }^{12}$ This test of every day memory problems takes 25 to 30 minutes to perform. Performance is measured as a screening score out of 12 . We have compared the memory scores of DM subjects with the raw data of the control group reported by Wilson et al. ${ }^{12}$ This control group consisted of 118 randomly selected subjects, aged between 16 and 69 years, with a mean IQ of 106 (range 68-136) established using NART and SPM scores. The IQ mean and ranges of $\omega$ our sample using these tests were very similar, that is, mean NART IQ of 106 (range 78-120) and mean SPM IQ 107.4 (range 65-126). Wilson $e t a l^{12}$ have shown inter-rater reliability to be $100 \%$. Validity has been established against other memory tests. In addition, validity has been established by comparison of scores against the observations by occupational therapist and physiotherapist of memory lapses, suggesting this is a valid test of everyday memory. ${ }^{12}$

More than one executive function task was included in order to incorporate the different components attributed to executive function. ${ }^{13}$ The three tests of executive function were: Wisconsin Card Sorting Test (WCST). ${ }^{14}$ The number of categories out of six were scored. Cognitive estimates test. ${ }^{15}$ Subjects have to guess the answer to a series of questions to which the exact answer is unlikely to be known, for example, how fast do race horses gallop?/what is the age of the oldest person in Britain today? Verbal fluency task. Subjects are required to name as many items as possible in a category in a given time. ${ }^{16}$ In this study the category animals was used allowing one minute to generate answers.

Because of the relationship between execu- $\widetilde{\sigma}$ tive test performance and intelligence, ${ }^{13}$ the $N$ DM subjects' scores on the three executive $\underset{\mathrm{N}}{\mathcal{N}}$ tasks were compared with an individually age and IQ matched control group (difference of $5 \mathrm{c}$ allowed on IQ or age) collected by PJM. Only 30 of our subjects were included because of the limits imposed by the tight matching.

(4) CTG repeat expansions were determined using Southern analysis. ${ }^{3}$ The CTG lengths of the DM cases examined in this study grouped $\stackrel{\square}{\square}$ to within the nearest $0.5 \mathrm{~kb}$ were: four subjects $(0-0.5 \mathrm{~kb}), 12$ subjects $(0.5-1 \mathrm{~kb})$, nine subjects 8 (1-1.5 kb), two subjects $(1.5-2 \mathrm{~kb})$, six subjects (2-2.5 kb), two subjects $(2.5-3 \mathrm{~kb})$, one subject $(4.5-5 \mathrm{~kb})$

(5) Statistical analysis was perfomed using the SPSS-PC software package (SPSS Inc, Chicago, IL). Many of the data were ordinal, so non-parametric statistics were used. 


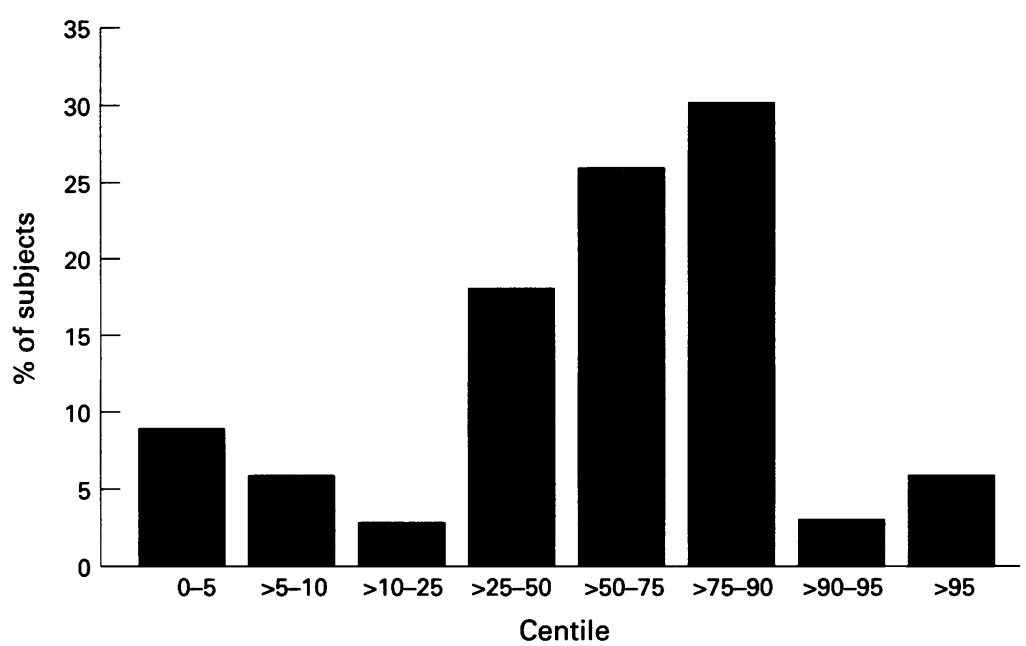

Figure 1 Standard Progressive Matrices scores in DM subjects in centile categories. The highest centile is associated with the highest intelligence score.

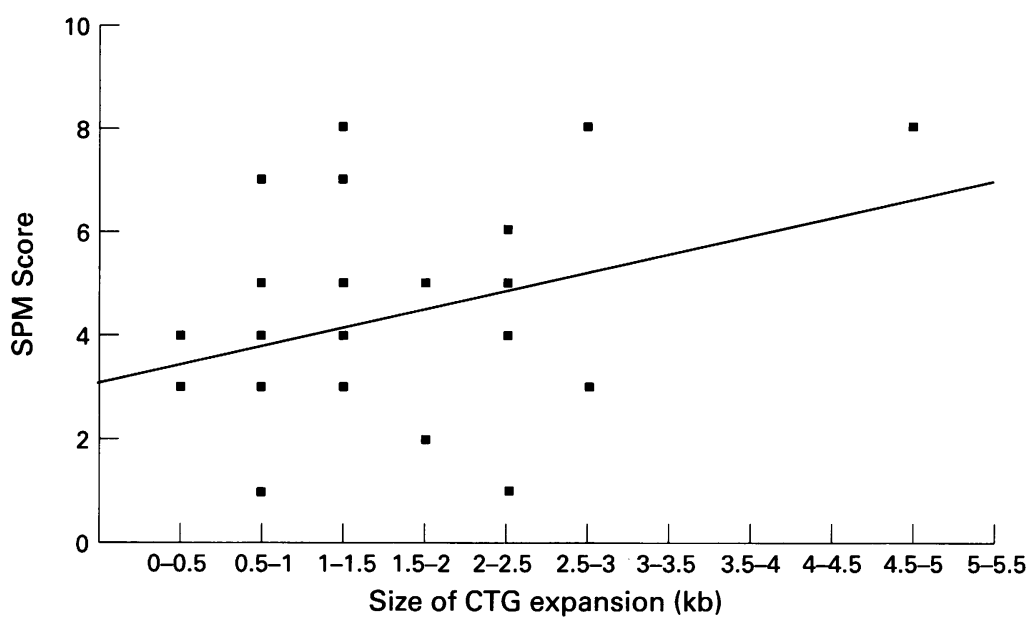

Figure 2 Standard Progressive Matrices (SPM) scores in DM subjects correlated with $C T G$ size. On the y axis SPM scores are numbered according to centile groups (that is, $8=0-5$ th centile, $7=>5-10$ th centile, $6=>10-25$ th centile, $5=>25-50$ th centile, $4=>50-75$ th centile, $3=>75-90$ th centile, $2=>90-95$ th centile, $1=>95$ th centile scores ). The highest centile is associated with the highest intelligence score.

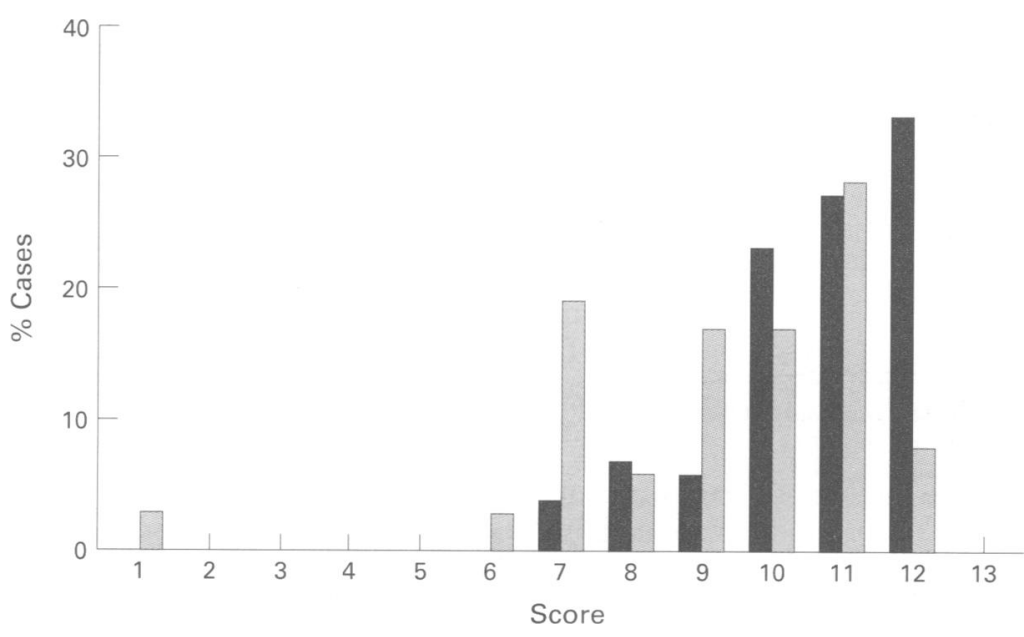

Figure 3 Rivermead Behavioural Memory Test scores in DM subjects (black bars) versus the controls (hatched bars) reported by Wilson et al. ${ }^{12}$ Scores reflect the number of items correct on the test. A score of 0-2 indicates severe impairment, 3-6 moderate impairment, and 7-9 poor memory.
The NART mean score was 106 (range 78-120). On the Standard Progressive Matrices (SPM), DM subjects performed surprisingly well: $25 / 36(69 \%)$ DM cases scored higher than the 50 th centile. Therefore the mean and median of this group is not below the average for the general population. Furthermore, there was no significant excess of DM subjects above the expected values at the 5 th or 10 th centile cutoffs (fig 1). The mean SPM score using conversion tables was 107.4. The score on the MMSE appeared to be within accepted clinical limits (a score of $<24$ reflects cognitive impairment). The scores of the DM subjects were 30 (13 cases), 29 ( 12 cases), 28 (four cases), 27 (five cases), 26 (one case), and 25 (one case).

When CTG length was correlated with standard progressive matrices scores (fig 2), a significant trend emerged $(R=0.38, p=0.024)$. However, analysis of these data suggested that the correlation was because of the subject with the largest expansion $(4.6 \mathrm{~kb})$, who had an SPM score below the 5th centile. When this subject was removed from the analysis, no correlation was apparent $(p=0.26)$ (fig 2$)$.

\section{MEMORY}

Although our DM group scored normally on tests of general intelligence, they showed a significant impairment of memory compared to controls (Mann Whitney U, p=0.0002) (fig 3). This difference remained significant (Mann Whitney $U, p=0.0003$ ) even when the subject with a score of 1 was excluded from the analysis. No significant correlation between CTG length and memory was observed. There was also no significant correlation between RBMT scores and length of illness (data not shown).

\section{EXECUTIVE FUNCTIONS}

DM subjects were compared with an IQ and age matched control group for executive function tests (fig 4). On the WCST the DM group showed a trend towards impairment (Mann Whitney $U, p=0.053$ ). The cognitive estimates test was significantly impaired in the DM subjects (Mann Whitney $U, p=0.047$ ). The verbal fluency task, using animals as a category, was not significantly different from normal subjects.

\section{Discussion}

We have investigated a group of milder DM cases and have not found any defect in general intelligence and only equivocal, mild executive function impairment. However, in the face of normal general intelligence, our results suggest that mild DM subjects as a group show impairment of memory function (results summarised in table 1).

The low range of the repeat sizes in all but one case in our series (childhood onset) confirms that abnormal IQ is not an important feature of the milder disease. Our data using the Standard Progressive Matrices Test ${ }^{10}$ and Mini-Mental State examination ${ }^{11}$ agree with an earlier study, ${ }^{17}$ which divided cases by disability and found that clinically mildly affected cases had normal IQs.
GENERAL INTELLIGENCE

This was assessed using three tests: the NART, SPM, and the MMSE. All subjects were alert during testing and found the tests stimulating.

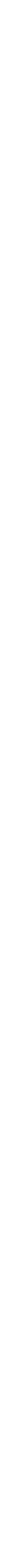



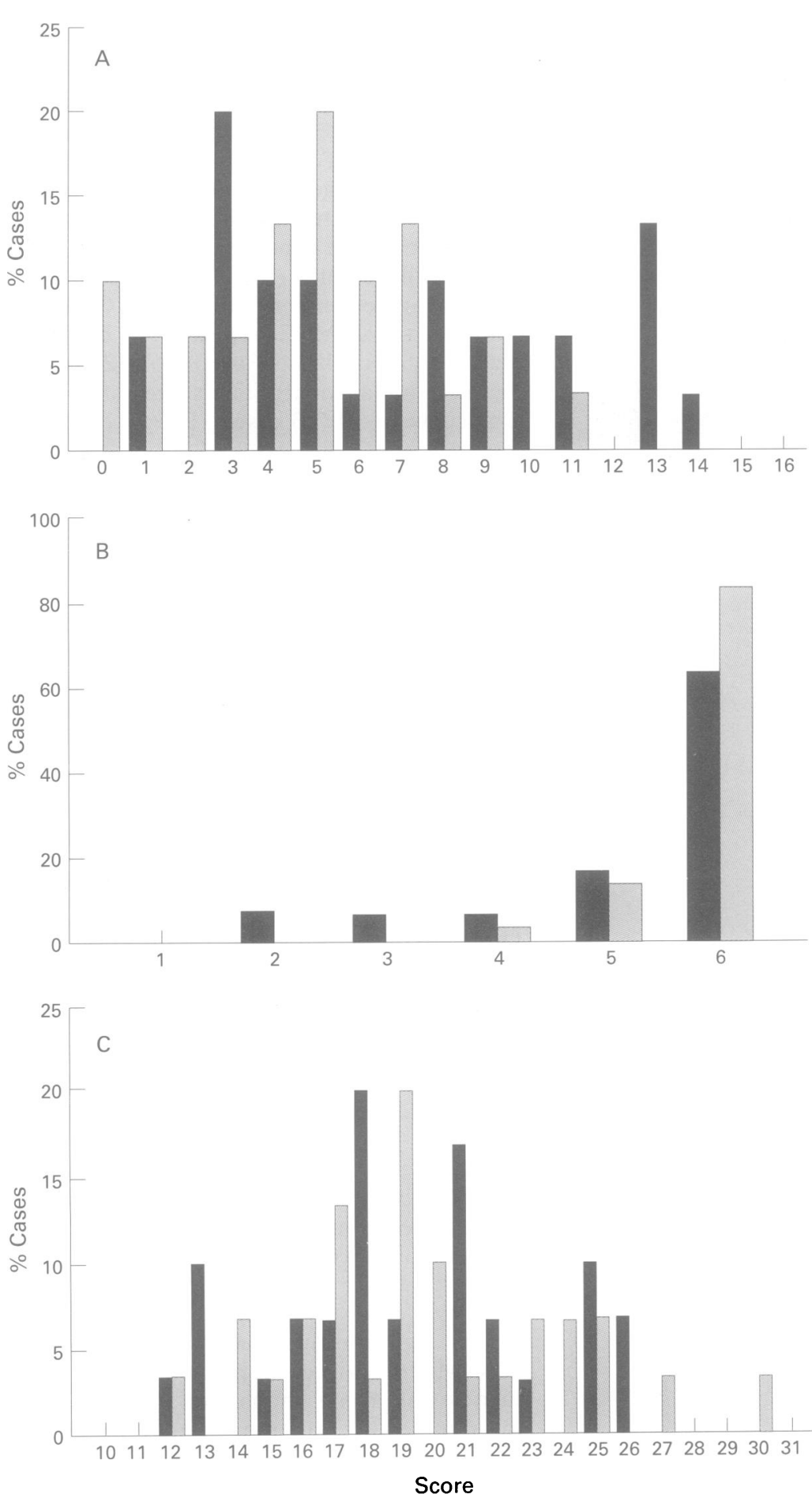

Figure 4 (A) Cognitive Estimates Test result in DM subjects (black bars) and IQ and age matched controls (hatched bars). Note that for this test a higher score reflects a worse performance. (B) Wisconsin Card Sorting Test result in DM subjects (black bars) and IQ and age matched controls (hatched bars). The score defines the numbers of categories achieved. (C) Verbal fluency task (using animal category) in 30 DM subjects (black bars) and age and $I Q$ matched controls (hatched bars). The score indicates the number of and age and $I Q$ matched controt

Our attempts to make correlations of CTG expansion size with cognitive function need to be interpreted with caution. The power to detect possible effects is compromised by our comparatively small sample size and because we have a relatively mild clinical group of patients. Our finding of a correlation of CTG repeat size with SPM score in our total sample is consistent with expectations from other published reports, although this has not been formally determined before. It is well known
Table 1 Summary of results (for full details see text and figures)

\begin{tabular}{ll}
\hline & Scores or $p$ values \\
\hline $\begin{array}{l}\text { General intellegence } \\
\text { NART (mean and range) }\end{array}$ & $106(78-120)$ \\
$\begin{array}{l}\text { SPM (mean and range)(WAIS } \\
\text { conversion scores) }\end{array}$ & $107.4(65-126)$ \\
MMSE & All scores $>24$ \\
Memory (DM v controls) & Mann Whitney U \\
RBMT & (MWU), $\mathrm{p}=0.0002$ \\
Executive function tests (DM $v$ & \\
$\quad$ controls) & \\
WCST & MWU, $\mathrm{p}=0.053$ \\
Cognitive estimates test & MWU, $\mathrm{p}=0.047$ \\
Verbal fluency & MWU, $\mathrm{p}=0.76$ \\
\hline
\end{tabular}

that congenital disease and early childhood onset cases are very often associated with mental retardation. A likely correlation is also expected based on the data of Hunter et $a l{ }^{2}$ which suggested an inverse relationship between the number of repeats and years in school. However, with the removal of the one subject with childhood onset and the longest CTG expansion in this series, the correlation between CTG repeat size and SPM scores was no longer significant. Since general cognition (SPM) was not impaired in our mild DM group as a whole, it is unlikely that CTG expansions in the smaller end of the disease size range are making a major impact on cognitive function.

Despite having normal general intelligence, our mild DM cases as a group exhibited a downward shift in the memory curve compared to normal controls. It is important to stress that this is a group effect and that the majority of the DM cases did not have abnormal memory. Wilson et $a l^{12}$ have suggested cut off points to help categorise memory disorders. Using these criteria one DM subject scored in the severely impaired range, one subject scored in the moderately impaired category (<7), and 15 subjects (out of 36 subjects tested) scored in the poor memory category $(7-9)$. These data imply that memory impairment in milder DM cases is disproportionate to IQ score.

The subject who scored 1 on the RBMT also met DSM IV criteria for dementia, aged 54. Before attributing this dementia to DM it is important to exclude other causes. The subject's family were not in favour of a more detailed examination and therefore this has not been possible.

Our data suggest that certain facets of executive function may be impaired in DM, since the cognitive estimates test and the Wisconsin card sorting test both showed trends towards impairment in DM, which were of borderline significance. These results need further investigation in a larger group of DM subjects, in order to be clear about the presence of an executive deficit. Nevertheless, our results contrast with those of Woodward et at that suggested that more than $70 \%$ of DM cases have executive function in the brain damaged range. Since this study was performed before the availability of genetic testing, congenital or childhood cases might have been included. 
In conclusion, these results show that mild DM patients have IQ scores similar to a normal population. Our data suggest that executive function may be impaired in these cases. However, as a group, there is a downward shift in memory function which suggests that this function may be more sensitive to small CTG repeat expansions than general cognition, as measured by the SPM test. Detailed analysis of cognitive function may allow better understanding of the pleiotropic effects of this mutation and provide starting points for the investigation of the molecular biology of these critical functions.

We thank all the participating subjects and their general practitioners, the generous assistance of the Medical Genetics tioners, the generous assistance of the Medical Genetics
Department, Addenbrooke's Hospital, and Mr D Dow, Mr J Hop, and Dr J Sussman. We especially thank Mrs R Paterson for Hon, and Dr J Sussman. We especially thank Mrs R Paterson for
secretarial assistance. This research project was undertaken during the research post on the Cambridge Psychiatric Training Scheme. Funding was received from the Lifespan Community NHS Trust and the Merck Lipha Research Fund Prize in Psychiatry.

1 Wieringa B. Commentary. Myotonic dystrophy is reviewed: back to the future? Hum Mol Genet 1994;3:1-7.

2 Hunter A, Tsilfidas C, Mettler G, et al. The correlation of age of onset with CTG trinucleotide repeat amplification in myotonic dystrophy. $\mathcal{f}$ Med Genet 1992;29:774-9.

3 Harley HG, Rundle SA, MacMillan JC, et al. Size of the unstable CTG repeat sequence in relation to phenotype and parental transmission in myotonic dystrophy. $A m \mathcal{F}$ Hum Genet 1993;52:1164-74.

4 Harper PS. Congenital myotonic dystrophy in Britain. Arch Dis Child 1975;50:505-15.

5 Woodward JB, Heaton RK, Simon DB, Ringel SP. Neuropsychological findings in myotonic dystrophy. $f$ Clin Neuropsychol 1982;4:335-42.

6 Portwood MM, Wicks JJ, Lieberman JAS, Duveneck MJ. Intellectual and cognitive function in adults with myotonic muscular dystrophy. Arch Phys Med Rehabil 1986;67:299303.

7 Chang L, Anderson T, Migneco OA, et al. Cerebral abnormalities in myotonic dystrophy. Arch Neurol 1993;50:91723.

8 Medical Research Council of the UK. Aids to the examination of the peripheral nervous system. Palo Alto: Pendragon House, 1978 .

9 Nelson HE. The national adult reading test (NART). Windsor: NFER-Nelson, 1982

10 Raven JC. Guide to the standard progressive matrices. London: Lewis and Co, 1960

11 Folstein MF, Folstein SE, McHugh PR. "Mini-Mental State". A practical method for grading the cognitive state of patients for the clinician. I Psychol Res 1975;12:189-98.

12 Wilson BC J, Baddeley A, Hiorns R. The development and validation of a test battery for detecting and monitoring everyday memory problems. 7 Clin Exp Neuropsychol 1989; 11:855-70.

13 Shallice T, Burgess PW, Frith CD. Can the neuropsychological case study approach be applied to schizophrenia. Psychol Med 1991;21:661-73.

14 Nelson HE. A modified card sorting test to frontal lobe defects. Cortex 1976;12:313-24.

15 Shallice T, Evans ME. The involvement of the frontal lobes in cognitive estimation. Cortex 1978;14:294-303.

16 Lezak MD. Neuropsychological assessment. 2nd ed. Oxford: Oxford University Press, 1983.

17 Bird TD, Follett C, Griep E. Cognitive and personality function in myotonic muscular dystrophy. $\mathcal{f}$ Neurol Neurosurg Psychiatry 1983;46:971-80. 